\title{
Radiation back reaction on moving branes
}

\author{
Ian G. Moss $⿴ 囗 十$ and James P Normant \\ School of Mathematics and Statistics, University of Newcastle Upon Tyne, NE1 7RU, UK
}

(Dated: December 9, 2018)

\begin{abstract}
This paper addresses the radiation back reaction problem for cosmological branes. A general framework is provided in which results are given for the radiation reaction with massles and massive scalar fields with flat extra dimensions and massless conformal fields in anti-de Sitter extra dimensions. For massless scalar field radiation the back reaction terms in the equation of motion are non-analytic. The interpretation of the radiation reaction terms is discussed and the equations of motion solved in simple cases. Nucleosynthesis bounds on dark radiation give a lower bound on the string vacuum energy scale of $\sqrt{A_{T}} m_{p}$, where $A_{T}$ is the tensor perturbation amplitude in the cosmic microwave background.
\end{abstract}

\section{INTRODUCTION}

Attempts to construct a unified theory of gravity and the other fundamental forces using superstrings have provided a new impetus for the study of higher dimensions and branes. In certain low energy limits, superstring theory reduces to a brane universe, or universes, where ordinary matter is confined to surfaces embedded in higher dimensions [1]. The cosmological evolution of these models can be different from the standard cosmological scenario [2, 3, 4] and their study is therefore worthwhile.

The matter fields on the brane interact with higher dimensional fields, which include the graviton and any particles associated with the graviton by supersymmetry. Cosmological equations, which describe the evolution from the point of view of the brane, can be obtained by decomposing the higher dimensional Einstein equations and applying boundary or junction conditions for the brane 2]. These equations reduce to the standard four dimensional equations for energies small compared to the vacuum energy scale of the brane.

Our interest here is mainly in the effects of radiation generated by the movement of the brane. We shall focus our attention on the radiation back reaction force on the brane. The radiation is closely related to the radiation due to a moving mirror [5]. The mirror, like the brane, disturbs the vacuum fluctuations of the radiation field through the effect of the boundary condition at the surface of the mirror. The vacuum fluctuations react back on the mirror though the pressure component in the stress energy tensor.

It may be useful to recall some of the old results on moving mirrors with massless scalar field radiation. The pressure force is proportional to the 3'rd time derivative of the mirror's position in two dimensions [5] and the 5'th time derivative in four dimensions $[\underline{6}]$. There is no radiation reaction force on a uniformly accelerating mirror. A rather perplexing result is that the pressure component of the stress energy tensor diverges as the distance from the mirror shrinks to zero. It is believed that the correct pressure on the mirror is obtained by dropping the divergent terms. Some support for this view is provided by the fact that the total work done by the divergent terms is zero, at least when the mirror motion has constant velocity asymptotically.

The moving mirror might also be placed in a heat bath with temperature $T$. In this case, for a mirror moving with a speed $v$, the thermal contribution to the pressure force is proportional to $T^{2} v$ in two dimensions [7] and $T^{4} v$ in four dimensions [8]. These agree with the forces which would be expected according to elementary kinetic theory applied to a mirror and a gas of photons.

The equation of motion for the mirror can be modified to take these radiation reaction forces into account. The modified equation of motion is analogous to the Abraham-Lorentz equation of motion for an electron with radiation damping [9], which offers a guide to the interpretation of the radiation reaction effects for the moving mirror and the moving brane. The Abraham-Lorentz equation for the electron's momentum is

$$
\frac{d p}{d t}=F+\tau \frac{d^{2} p}{d t^{2}}
$$

where $F$ is an external force and $\tau=2 e^{2} / 3 m c^{3}$. A feature of this equation is the existence of 'runaway' solutions $p \propto \exp (t / \tau)$, which are rejected on the grounds that the radiation reaction term should be only a small correction to

*Electronic address: ian.moss@ncl.ac.uk

${ }^{\dagger}$ Electronic address: j.p.norman@ncl.ac.uk 
the equation of motion. The runaway solutions can be eliminated by replacing the equation by an integrodifferential equation, but this introduces another feature, 'preacceleration', where the electron begins to respond a short time before the external force is applied. We shall see that these features can be found in the brane system.

Recent work related to radiating branes includes [10], which examined the radiation due to a uniformly accelerated brane. The authors did not consider the radiation reaction, but it would be expected to vanish for this type of motion. The brane can also radiate though interactions of the type $X+X \rightarrow Y$, where the $X$ 's are particles of matter and $Y$ is a bulk graviton. The effect of these gravitons on the brane persists, and resembles ordinary radiation. In some very specific models of brane cosmology, the gravitons can make a small contribution to the expansion rate during the nucleosynthesis era. This has been investigated by a number of authors [11, 12, 13]. A similar type of reaction, where $X$ is the inflaton and $Y$ a bulk scalar, could also change the nature of reheating after a period of inflation [14] .

The next section contains some general results for the radiation from moving branes and mirrors. We take some care with the perturbation theory because we would like to ensure that the calculations give the vacuum expectation value $\left\langle\right.$ in $\left|T_{a b}\right|$ in $\rangle$ of the stress energy tensor rather than $\left\langle\right.$ out $\left|T_{a b}\right|$ in $\rangle$. It is also important to separate the radiation reaction calculation from effective action and casimir energy calculations. From the point of view of the brane and its cosmological evolution, the radiation back reaction effect is a dissipative phenomenon and cannot be derived from a reduced effective action which only depends on the degrees of freedom attached to the brane. However, we shall see that the Schwinger-Keldeysh formalism gives a reduced effective action which can be used to analyse both dissipative and non-dissipative effects.

\section{RADIATION BACK REACTION}

\section{A. Backreaction equations}

Our approach to the back reaction on a brane moving in one extra dimension is closely based on the back reaction problem for a moving mirror [6]. In both cases the radiation is produced because the radiation satisfies boundary conditions on a moving boundary. In moving mirror problems the back reaction force is determined by the pressure components of the expectation value of the stress energy tensor.

The back reaction of this radiation on the motion of a brane in five dimensions can be obtained by reducing the five dimensional Einstein equations in the manner described by Shiromizu, Maeda and Sasaki [2]. This reduction assumes a reflection symmetry between to two sides of the brane. For the 5-dimensional Einstein equations we have

$$
G_{a b}=\kappa_{5}^{2} T_{a b}
$$

For simplicity, we shall consider just a 5 -dimensional cosmological constant $\Lambda_{5}$, a brane vacuum energy $\lambda$ and 5 dimensional radiation,

$$
T_{a b}=-\kappa_{5}^{-2} \Lambda_{5} g_{a b}-\lambda h_{a b} \delta(\Sigma)+\left\langle\operatorname{in}\left|T_{a b}^{r}\right| \operatorname{in}\right\rangle
$$

where $h_{a b}$ is the metric induced on the brane by the 5-dimensional metric $g_{a b}$. Using the Gauss-Codacci equations, Shiromizu, Maeda and Sasaki show that the Einstein tensor ${ }^{(4)} G_{\mu \nu}$ satisfies

$$
{ }^{(4)} G_{\mu \nu}+\Lambda h_{\mu \nu}=\frac{2 \kappa_{5}^{2}}{3}\left\langle\text { in }\left|T_{\mu \nu}^{r}+\left(T_{n n}^{r}-\frac{1}{4} T^{r}\right) h_{\mu \nu}\right| \text { in }\right\rangle-E_{\mu \nu}
$$

where $E_{\mu \nu}$ is a projection of the Weyl tensor, $C_{n \mu n \nu}$ and therefore trace-free. The 4-dimensional cosmological constant term and Newton's constant are related to their 5-dimensional counterparts by

$$
\begin{aligned}
\Lambda & =\frac{1}{12}\left(6 \Lambda_{5}+\kappa_{5}^{4} \lambda^{2}\right) \\
G & =\frac{\kappa_{5}^{4} \lambda}{48 \pi}
\end{aligned}
$$

In order to recover the correct low energy limit for the single brane we require $\lambda>0$ and, for a small or vanishing cosmological constant, $\Lambda_{5}<0$.

The $E_{\mu \nu}$ term can be eliminated by taking the trace of (4), giving an equation for the Ricci scalar of the brane,

$$
{ }^{(4)} R=4 \Lambda-2 \kappa_{5}^{2}\left\langle\text { in }\left|T_{n n}^{r}\right| \text { in }\right\rangle
$$

In a cosmological context, this is the equation of motion of the scale factor. The expectation value on the right includes the radiation damping effect on the expansion rate of the universe. In general, the expectation value depends 
on both the five dimensional metric and the brane motion. However, in this paper we shall assume that the back reaction of the radiation on the five dimensional metric can be neglected in the quantum calculation.

For a spatially flat, homogeneous universe with scale factor $a(t)$ and expansion rate $H(t)$, the cosmological evolution equation (7) becomes

$$
6 \dot{H}+12 H^{2}=4 \Lambda-2 \kappa_{5}^{2}\left\langle\text { in }\left|T_{n n}^{r}\right| \text { in }\right\rangle
$$

The initial condition as $t \rightarrow-\infty$ is provided by the Friedman equation $3 H^{2}=\Lambda$. The Friedman equation is modified at all other times by the Weyl term,

$$
E_{00}=\frac{2 \kappa_{5}^{2}}{3}\left\langle\operatorname{in}\left|\left(T_{00}^{r}-T_{n n}^{r}\right)\right| \text { in }\right\rangle+\Lambda-3 H^{2}
$$

which can be interpreted as energy lost by the brane. Note, however, that the quantum term can be negative.

Typically, the radiation reaction $\left\langle\right.$ in $\left|T_{n n}^{r}\right|$ in $\rangle$ will only be significant for very early times, but it leaves behind a residual 'dark radiation' term [15] obtained by integrating (8),

$$
E_{00} \sim \frac{2 \kappa_{5}^{2}}{a^{4}} \int_{-\infty}^{\infty} H\left(t^{\prime}\right) a\left(t^{\prime}\right)^{4}\left\langle\operatorname{in}\left|T_{n n}^{r}\left(t^{\prime}\right)\right| \text { in }\right\rangle d t^{\prime}
$$

In principle, this term behaves like a contribution to the radiation energy density, but we have not, so far, considered the effects of ordinary matter. If, for example, matter is generated by reheating after a period of inflation, then the dark radiation produced during or prior to inflation would be inflated away [16].

\section{B. Quantum stress energy tensor}

We shall obtain some general results for the expectation value of the stress energy tensor using perturbation theory. The classical action of the radiation field $\phi$ is

$$
S_{r}=\frac{1}{2} \int_{\mathcal{M}} \phi \Delta \phi d v
$$

where $\Delta$ is a second order operator and $d v$ is the volume measure. The unperturbed system is chosen to be in vacuum state where the green functions of the radiation with the prescribed boundary conditions are known explicitly. For scalar fields, the boundary conditions can be Dirichlet, where the field vanishes on the boundary, or Robin, where the normal derivatives of the field are prescribed. Our notation for Green functions follows ref. [17].

The relation between the perturbed and unperturbed boundaries can be defined by a diffeomorphism $f$ as shown in figure 1 If the perturbation is small, then the vacuum state should be invariant. Expectation values calculated for the brane $\Sigma$ and the metric $g$ should be equivalent to the expectation values calculated with the unperturbed brane $f^{-1}(\Sigma)$ and the metric $f^{*} g$. By this means we can replace a perturbation of the brane by a perturbation of the metric.

The number of dimensions $n$ is left arbitrary. Local coordinates can be adapted to the unperturbed brane so that $n-1$ coordinates $x^{\mu}$ describe the position on the brane and a coordinate $z=-x^{n}$ describes the distance to the perturbed brane. The perturbed brane is then described by the function $z\left(x^{\mu}\right)$.

We begin with the Wightman functions $G^{ \pm}$of the scalar field $\phi$, defined by

$$
\begin{aligned}
& G^{+}\left(x . x^{\prime}\right)=\left\langle\operatorname{in}\left|\phi(x) \phi\left(x^{\prime}\right)\right| \text { in }\right\rangle \\
& G^{-}\left(x . x^{\prime}\right)=\left\langle\operatorname{in}\left|\phi\left(x^{\prime}\right) \phi(x)\right| \text { in }\right\rangle .
\end{aligned}
$$

The Wightman functions satisfy the homogeneous wave equation

$$
\Delta G^{ \pm}=0
$$

Their sum defines the Hadamard function,

$$
G^{(1)}=G^{+}+G^{-}
$$

The change in Hadamard's function when the operator is perturbed by the moving brane is

$$
\delta G^{(1)}=-G_{R}(\delta \Delta) G^{(1)}-G^{(1)}(\delta \Delta) G_{A}
$$




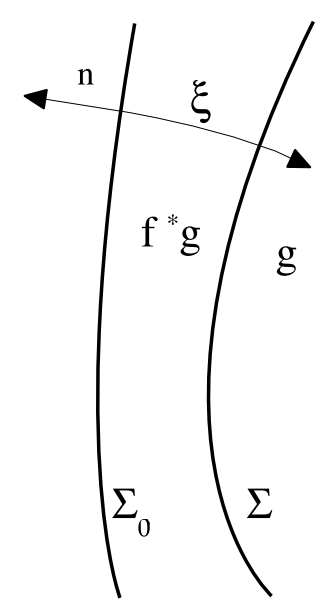

FIG. 1: The brane $\Sigma$ can be obtained by mapping the unperturbed brane $\Sigma_{0}$ along the intergral curves of a vector field $\xi$. The mapping is denoted by $f$ and the pullback mapping by $f^{*}$.

where $G_{R}$ and $G_{A}$ are the retarded and advanced propagators,

$$
G_{R}\left(x, x^{\prime}\right)=G_{A}\left(x^{\prime}, x\right)=-i\left\langle\operatorname{in}\left|\left[\phi(x) \phi\left(x^{\prime}\right)\right]\right| \operatorname{in}\right\rangle \theta\left(t-t^{\prime}\right)
$$

The perturbed form of the Hadamard function is real, symmetric and causal.

The first order change in the Hadamard function can be cast into a form which is more convenient for calculations,

$$
\delta G^{(1)}=-4 \operatorname{Im}\left(G_{0}^{>}(\delta \Delta) G_{0}^{<}\right)
$$

where the subscript denotes the use of the unperturbed green function, and

$$
G_{0}^{>}\left(x, x^{\prime}\right)=G_{0}^{<}\left(x^{\prime}, x\right)=i G_{0}^{+}\left(x, x^{\prime}\right) \theta\left(t-t^{\prime}\right)
$$

The subscripts will subsequently be dropped.

The next step is to find an expression for $\delta \Delta$. Consider the general second order operator

$$
\Delta=-\nabla^{2}+\xi R+m^{2}
$$

The change in the operator is induced by the coordinate transformation $\xi^{a}=-z\left(x^{\mu}\right) n^{a}$ as shown in figure 1 The diffeomorphism invariance of the classical action of the radiation (11) implies that

$$
\int_{\mathcal{M}}\left(\phi(\delta \Delta) \phi+\left(\xi^{a} \nabla_{a} \phi\right) \Delta \phi+\phi \Delta\left(\xi^{a} \nabla_{a} \phi\right)\right) d v+\int_{\Sigma} z \phi \Delta \phi d v=0
$$

After integration by parts we deduce that

$$
\delta \Delta=\nabla_{a} \xi^{a} \Delta-\Delta \xi^{a} \nabla_{a}
$$

The required combination of green functions can now be found explicitly by a covariant volume integral,

$$
\delta G^{(1)}=4 \operatorname{Im} \int_{\mathcal{M}} G^{>}\left(x, x^{\prime}\right)\left(\Delta \xi^{a^{\prime}} \nabla_{a^{\prime}}-\nabla_{a^{\prime}} \xi^{a^{\prime}} \Delta\right) G^{>}\left(y, x^{\prime}\right) d v^{\prime}
$$

The imaginary part of the integrand consists of a total divergence which reduces by the divergence theorem to

$$
\delta G^{(1)}=4 \operatorname{Im} \int_{\Sigma}\left(\nabla_{n^{\prime}} G^{>}\left(x, x^{\prime}\right) \nabla_{n^{\prime}} G^{>}\left(y, x^{\prime}\right)-G^{>}\left(x, x^{\prime}\right) \nabla_{n^{\prime}}^{2} G^{>}\left(y, x^{\prime}\right)\right) z\left(x^{\prime}\right) d v^{\prime}
$$

The reason for obtaining this expression for the Hadamard function is that it can be used to find the expectation value of the stress energy tensor. The expectation value of the stress energy tensor contains terms such as

$$
\left\langle\operatorname{in}\left|\left(\nabla_{a} \phi \nabla_{b} \phi+\nabla_{b} \phi \nabla_{a} \phi\right)\right| \text { in }\right\rangle,
$$


which can be evaluated by applying an operator to the hadamard function and taking a coincidence limit [17],

$$
\left[\nabla_{a} \nabla_{b^{\prime}} G^{(1)}\right]
$$

The brackets denote the coincidence limit $x=x^{\prime}$. The full stress energy tensor is given by

$$
\left\langle\text { in }\left|T_{a b}^{r}\right| \text { in }\right\rangle=\frac{1}{2}\left[D_{a^{\prime} b} G^{(1)}\right]
$$

where the distribution valued operator $D_{a b}$ is given by

$$
D_{a b}=\frac{1}{2} \frac{\delta^{2}}{\delta \phi^{2}} T_{a b}=\frac{\delta \Delta}{\delta g^{a b}} .
$$

In the minimal case, for example,

$$
D_{a b}\left(x, x^{\prime}\right)=\left(\nabla_{a^{\prime}} \nabla_{b}-\frac{1}{2} g_{a b} g^{c d} \nabla_{c^{\prime}} \nabla_{d}\right) \delta(y, x) \delta\left(y, x^{\prime}\right)
$$

For Dirichlet boundary conditions, the change in the normal component of the stress energy tensor at the brane position is given by (24),

$$
\delta\left\langle\operatorname{in}\left|T_{n n}^{r}\right| \text { in }\right\rangle=\operatorname{Im} \int_{\Sigma}\left(\nabla_{n} \nabla_{n^{\prime}} G^{>}\left(x, x^{\prime}\right)\right)^{2} z\left(x^{\prime}\right) d v^{\prime}
$$

which is equivalent to an old result due to Ford and Vilenkin [6]. For a moving mirror, this stress energy component generates the pressure force on the mirror. Since the unperturbed value of the stress energy does not affect the motion we shall drop the $\delta$ and only keep the perturbed value from now on.

\section{Schwinger-Keldeysh version}

Before proceeding, it is interesting to see how the same results can be obtained more directly from an effective action using Schwinger-Keldeysh methods [18, 19, 20]. In this approach, the operator $\Delta$ is a $2 \times 2$ matrix which depends on two copies of the background fields,

$$
\Delta_{S K}=\left(\begin{array}{cc}
\Delta\left[g_{1}\right] & 0 \\
0 & \Delta\left[g_{2}\right]
\end{array}\right)
$$

The radiation reaction forces can be obtained by variation of the one loop correction to the Schwinger-Keldeysh effective action,

$$
W_{S K}=-\frac{i}{2} \log \operatorname{det}\left(i G_{S K}\right)
$$

where $G_{S K}$ is the green function in the Schwinger-Keldeysh approach. Let $S\left[g_{1}\right]$ be the classical gravitational action, then $\delta_{1} S+\delta_{1} W_{S K}=0$, where $\delta_{1}$ denotes variation of $g_{1}$, and $g=g_{1}=g_{2}$ after the variation.

The Schwinger-Keldeysh green function is a $2 \times 2$ matrix

$$
G_{S K}=\left(\begin{array}{cc}
G_{T} & i G^{-} \\
-i G^{+} & -G_{\bar{T}}
\end{array}\right)
$$

where

$$
\begin{aligned}
G_{T}\left(x, x^{\prime}\right) & =i G^{+} \theta\left(t-t^{\prime}\right)+i G^{-} \theta\left(t^{\prime}-t\right) \\
G_{\bar{T}}\left(x . x^{\prime}\right) & =i G^{-} \theta\left(t-t^{\prime}\right)+i G^{+} \theta\left(t^{\prime}-t\right) .
\end{aligned}
$$

(Note that $G_{T}$ is identical to the Feynman Green function if the in and out vacua are the same state, but not necessarily identical otherwise). The Schwinger-Keldeysh Green function changes under perturbations of the operator by

$$
\delta G_{S K}=-G_{S K}(\delta \Delta)_{S K} G_{S K}
$$


Multiplying out the matrices gives, for example,

$$
\delta G_{T}=-G_{T}(\delta \Delta) G_{T}+G^{-}(\delta \Delta) G^{+}
$$

Under the same restrictions as before, this is equivalent to

$$
\delta G_{T}=-2 \operatorname{Im}\left(G^{>}(\delta \Delta) G^{<}\right)
$$

The variation of the Schwinger-Keldeysh effective action to first order in perturbation theory is therefore

$$
\delta_{1} W_{S K}=\operatorname{Im} \operatorname{tr}\left(\left(\delta_{1} \Delta\right) G^{>}(\delta \Delta) G^{<}\right) .
$$

By (27) and (28), this is equivalent to the previous result for the stress energy tensor of the radiation.

Alternatively, we we can regard the action as a function of the brane position and obtain a reduced action formulation, often called the moduli space approximation. Let

$$
W[z, g]=\frac{1}{2} \operatorname{Im} \operatorname{tr}\left(\delta \Delta G^{>} \delta \Delta G^{<}\right) .
$$

Variation with respect to $z$ can be related to a metric variation $\delta_{1} W$ by diffeomorphism invariance, as in figure 1 The effective equations of motion are then

$$
\frac{\delta S}{\delta z}+\frac{\delta W}{\delta z}=0
$$

The Schwinger-Keldeysh formalism is usually applied to non-equilibrium thermal field theory, using finite temperature version of the propagator (33). Our results are therefore equally applicable to finite temperatures (for a time-independent background) if we replace the function $G^{>}\left(x, x^{\prime}\right)$ by the finite temperature version.

\section{BRANES MOVING IN FLAT SPACE}

We shall examine the radiation back reaction force on the brane to leading order in the displacement from a flat hyperplane in flat space. Consider the massless field with Dirichlet boundary conditions to begin with. The unperturbed green function (19) can be expressed in terms of basis functions which vanish at $z=0$,

$$
G^{>}\left(x, x^{\prime}\right)=\int_{0}^{\infty} \frac{d q}{2 \pi} \int \frac{d^{n-2} k}{(2 \pi)^{n-2}} \frac{i}{2 \omega} 4 \sin q z \sin q z^{\prime} e^{i \mathbf{k}\left(\mathbf{x}-\mathbf{x}^{\prime}\right)-i \omega\left(t-t^{\prime}\right)} \theta\left(t-t^{\prime}\right)
$$

where $\omega=\left(k^{2}+q^{2}\right)^{1 / 2}$. Inserting this into equation (30) for the stress energy tensor gives

$$
\left\langle\text { in }\left|T_{n n}^{r}\right| \text { in }\right\rangle=\operatorname{Im} \operatorname{reg} \int_{-\infty}^{\infty} \frac{d q}{2 \pi} \frac{d q^{\prime}}{2 \pi} \int \frac{d^{n-2} k}{(2 \pi)^{n-2}} \frac{q^{2} q^{\prime 2}}{\omega \omega^{\prime}} I[z]
$$

where 'reg' indicates some form of regularisation has been performed to make the integral finite and

$$
I[z]=\int_{-\infty}^{t} e^{-i\left(\omega+\omega^{\prime}\right)\left(t-t^{\prime}\right)} z\left(t^{\prime}\right) d t^{\prime}=-i\left(\left(\omega+\omega^{\prime}\right)-i \partial_{t}\right)^{-1} z
$$

Note the importance of taking the imaginary part of the expression after regularisation. It is useful to introduce

$$
F_{n}(x)=\operatorname{reg} \int \frac{d q}{2 \pi} \frac{d q^{\prime}}{2 \pi} \frac{d^{n-2} k}{(2 \pi)^{n-2}} \frac{q^{2} q^{\prime 2}}{\omega \omega^{\prime}} \frac{-i}{\left(\omega+\omega^{\prime}\right)-i x}
$$

and then

$$
\left\langle\text { in }\left|T_{n n}^{r}\right| \text { in }\right\rangle=\operatorname{Im} F_{n}\left(\partial_{t}\right) z
$$

The integral diverges for all values of the dimension $n$ which rules out dimensional regularisation. However, an analytic regularisation scheme can be used where we define

$$
F_{n s}(x)=\frac{i}{\Gamma(s+1)} \int_{0}^{\infty} d \lambda \lambda^{s} \int \frac{d q}{2 \pi} \frac{d q^{\prime}}{2 \pi} \frac{d^{n-2} k}{(2 \pi)^{n-2}} \frac{-q^{2} q^{\prime 2}}{\omega \omega^{\prime}} e^{-\lambda\left(\omega+\omega^{\prime}\right)+i \lambda x}
$$


and take the value at $s=0$, removing pole terms if necessary. After integrating over $q$, we have

$$
F_{n s}(x)=\frac{-4 i}{\Gamma(s+1)} \int_{0}^{\infty} d \lambda \lambda^{s} \int \frac{d^{n-2} k}{(2 \pi)^{n-2}}(\lambda k)^{-2} K_{1}(\lambda k)^{2} e^{i \lambda x}
$$

where $K_{1}$ is a Bessel function of the second kind. The remaining integrals give

$$
F_{n s}(x)=\frac{1}{\Gamma(s+1)} \frac{x^{n-s+1}}{2 \pi^{n / 2}} \frac{\Gamma(n / 2)^{3}}{(n+1) \Gamma(n)^{2}} \frac{i^{n-s}}{\sin \pi(n-s)}
$$

After removing the pole at $s=0$, the regularised expression is

$$
F_{n}(x)=-\frac{(-i x)^{n+1}}{4 \pi^{n / 2}}\left(1-i \ln \left(\frac{x}{\mu}\right)\right) \frac{\Gamma(n / 2)^{3}}{(n+1) \Gamma(n)^{2}}
$$

where $\mu$ is a renormalisation constant.

Some examples are

$$
\begin{aligned}
\operatorname{Im} F_{2}(x) & =-\frac{x^{3}}{12 \pi} \\
\operatorname{Im} F_{4}(x) & =\frac{x^{5}}{720 \pi^{2}} \\
\operatorname{Im} F_{5}(x) & =-\frac{x^{6}}{16384 \pi^{2}} \ln \left(\frac{x}{\mu}\right)
\end{aligned}
$$

The results for two and four dimensions agree with those found by Ford and Vilenkin for the moving mirror problem 6].

The dependence on $\mu$ in odd dimensions can be traced to need for new counterterms in the classical action [21] which arise from the $n$ 'th heat kernel coefficient of the operator $\Delta$ [22]. In five dimensions, the heat kernel coefficient contains boundary terms of the form

$$
S_{5 s}=\frac{1}{s} \int_{\Sigma} \nabla_{\mu} K_{\nu \rho} \nabla^{\mu} K^{\nu \rho} d v
$$

and other permutations of the indices, where $K$ is the extrinsic curvature of the brane. These counterterms produce a $\partial_{t}{ }^{6} z$ in the equation of motion (since $K=-\partial_{t}{ }^{2} z$ to leading order) which cancels the divergence in the radiation reaction and leaves the logarithmic dependence on $\mu$. How the logarithmic derivatives are interpreted, and how to solve the equations of motion for the brane will be covered in section 4 .

The radiation back reaction from a massive scalar field with mass $m$ can be found by redefining $\omega=\left(k^{2}+q^{2}+m^{2}\right)^{1 / 2}$ in eq. (47). In even dimensions, the integral results in

$$
\operatorname{Im} F_{n}(x)=\frac{1}{8(4 \pi)^{n / 2}} m^{n-1} x^{2} \Gamma\left(\frac{1-n}{2}\right) \Gamma\left(\frac{1}{2}\right){ }_{2} F_{1}\left(\frac{1-n}{2}, \frac{1}{2} ; 2 ;-\frac{x^{2}}{4 m^{2}}\right)
$$

where ${ }_{2} F_{1}$ is a hypergeometric function. In odd dimensions, we discard the pole term and retain only the finite part of the same expression.

For small values of $x, F_{n}$ is of order $x^{2}$. If the position of the brane is oscillating with a frequency $\omega<m$, the radiation reaction force is proportional to $\omega^{2}$, which is similar to a known result for a domain wall in four dimensions [23]. For large values of $x$, the hypergeometric functions have branch cuts in the region $|x|>2 m$ and logarithmic terms appear in the large $x$ limits for both even and odd dimensions. The leading terms agree with the massless results given above.

\section{BRANES MOVING IN ANTI-DE SITTER SPACE}

Branes in anti-de Sitter space are interesting from a cosmological point of view. The intrinsic geometry of a homogeneous brane moving in anti-de Sitter space is similar to a cosmological model [24, 25]. The effects of the higher dimensional cosmological constant and the vacuum energy on the brane can be fine-tuned to give a relatively small effective cosmological constant on the brane [26, 27].

As before, we will neglect the back reaction of the motion of the brane on the bulk metric when calculating the quantum stress energy tensor. We shall consider small perturbations of a single flat brane whose penrose diagram is 

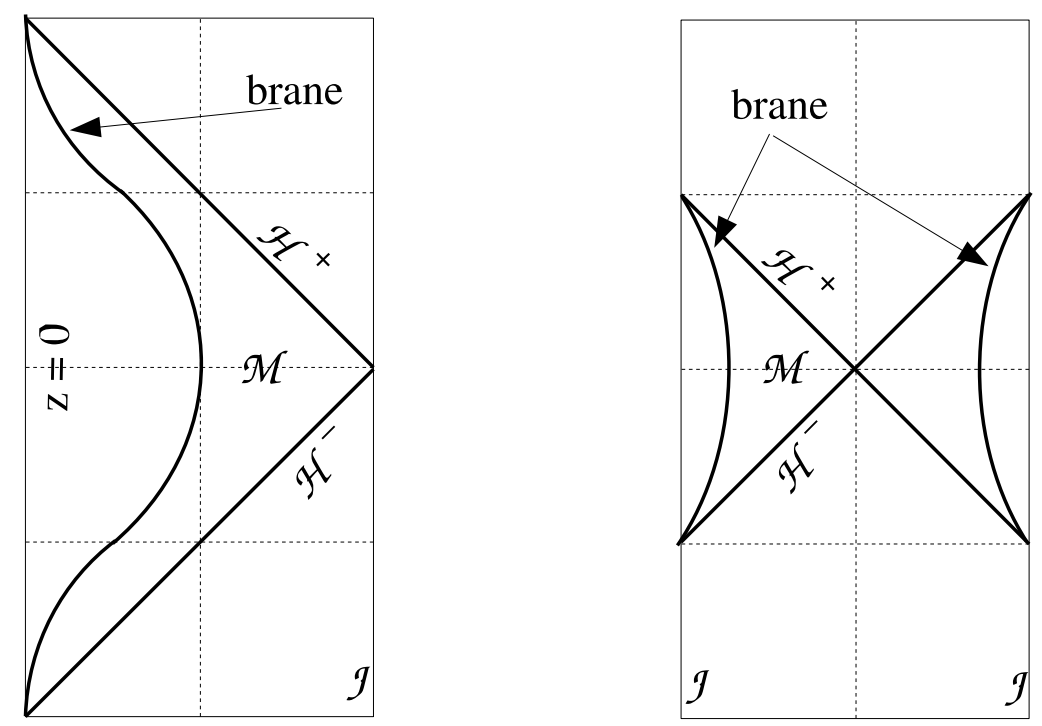

FIG. 2: On the left is a Penrose diagram of universal anti-de Sitter space showing the location of a timelike slice of the flat brane. The coordinate system extends on the right of the brane $(z>\sigma)$ to the horizons $\mathcal{H}^{+}$and $\mathcal{H}^{-}$where the timelike killing vector becomes null. On the right is a copy of the Penrose diagram to show the location of a de Sitter space brane.

shown in figure 2 We take the vacuum state related to the timelike translation symmetry along the brane, which we call the brane vacuum.

The brane vacuum has the disadvantage that there are horizons where the timelike killing field vanishes. The horizons can be avoided, for example by considering a two brane system and taking the limit where the separation between the branes becomes large. If, instead, we use a vacuum state which covers the whole of anti-de Sitter space, then we have to consider the effects of Bogolubov coefficients [17]. In the case of the de Sitter brane, the Euclidean vacuum state can be used 21]. The Euclidean vacuum becomes a thermal state with respect to the brane, creating additional thermal effects in the radiation reaction force. We shall not consider this further here.

One of the many ways to express the Anti-de Sitter metric in $n$ dimensions is the conformally flat form,

$$
\sigma^{2} z^{-2}\left(d z^{2}+\eta_{\mu \nu} d x^{\mu} d x^{\nu}\right)
$$

with Minkowski metric $\eta_{\mu \nu}$. The Anti-de Sitter radius $\sigma$ is related to the cosmological constant in $n$ dimensions by

$$
\Lambda_{n}=-\frac{(n-1)(n-2)}{2 \sigma^{2}} .
$$

The unperturbed brane will be placed at $z=\sigma$ and the manifold extends in the $z>\sigma$ direction only. The covering space can be filled in by reflection symmetry about the brane.

The Green functions can be obtained from the normalised modes $u_{k q}$ of the scalar wave equation. These are

$$
u_{k q}=\frac{1}{(2 \omega)^{1 / 2}}\left(\frac{z}{\sigma}\right)^{(n-1) / 2} H_{\nu}(q, z) e^{i(\mathbf{k} \cdot \mathbf{x}-\omega t)}
$$

with $\omega=\left(k^{2}+q^{2}\right)^{1 / 2}$ and $H_{\nu}$ a combination of Hankel functions of order

$$
\nu^{2}=\frac{1}{4}+\sigma^{2}\left((\xi-\xi(n)) R+m^{2}\right) .
$$

Conformal curvature coupling corresponds to the case $\xi=\xi(n)$ and $\nu=1 / 2$.

For modes which vanish on the brane,

$$
H_{\nu}(q, z)=\frac{i(\pi q \sigma)^{1 / 2}}{2\left|H_{\nu}^{(1)}(q \sigma)\right|}\left(H_{\nu}^{(1)}(q z) H_{\nu}^{(2)}(q \sigma)-H_{\nu}^{(2)}(q z) H_{\nu}^{(1)}(q \sigma)\right)
$$

Note that the normal derivatives of the mode functions when evaluated on the brane $z=\sigma$ are then given by

$$
\nabla_{n} u_{k q}=\frac{1}{(2 \omega)^{1 / 2}}\left(\frac{2 q \sigma}{\pi}\right)^{1 / 2} \frac{2^{1 / 2} q}{\left|H_{\nu}^{(1)}(q \sigma)\right|} e^{i(\mathbf{k} \cdot \mathbf{x}-\omega t)}
$$


In the conformal case $\nu=1 / 2$, this reduces to

$$
\nabla_{n} u_{k q}=\frac{1}{(2 \omega)^{1 / 2}} 2^{1 / 2} q e^{i(\mathbf{k} \cdot \mathbf{x}-\omega t)}
$$

which is also the value obtained in flat space.

The normal derivatives of the Wightman function are needed to obtain the stress energy tensor. The Wightman function can be obtained from a mode sum,

$$
G^{+}\left(x, x^{\prime}\right)=\int_{0}^{\infty} \frac{d q}{2 \pi} \int \frac{d^{n-2} k}{(2 \pi)^{n-2}} u_{k q}(x) u_{k q}^{*}\left(x^{\prime}\right)
$$

The normal derivatives at the brane can be expressed as

$$
\nabla_{n} \nabla_{n^{\prime}} G^{+}\left(x, x^{\prime}\right)=\int_{0}^{\infty} \frac{d q}{2 \pi} \int \frac{d^{n-2} k}{(2 \pi)^{n-2}} \frac{2 q^{2}}{2 \omega}\left|\frac{H_{1 / 2}^{(1)}(q \sigma)}{H_{\nu}^{(1)}(q \sigma)}\right|^{2} e^{i\left(\mathbf{k} \cdot\left(\mathbf{x}-\mathbf{x}^{\prime}\right)-\omega\left(t-t^{\prime}\right)\right)}
$$

As in the flat space example, the expectation value of the normal components of the stress energy tensor are given in terms of an integral by

$$
\left\langle\operatorname{in}\left|T_{n n}^{r}\right| \text { in }\right\rangle=\operatorname{Im} F_{n}\left(\partial_{t}\right) z
$$

where the anti-De Sitter space version of $F$ is

$$
F_{n}(x)=\operatorname{reg} \int \frac{d q}{2 \pi} \frac{d q^{\prime}}{2 \pi} \frac{d^{n-2} k}{(2 \pi)^{n-2}} \frac{q^{2} q^{\prime 2}}{\omega \omega^{\prime}}\left|\frac{H_{1 / 2}^{(1)}(q \sigma) H_{1 / 2}^{(1)}\left(q^{\prime} \sigma\right)}{H_{\nu}^{(1)}(q \sigma) H_{\nu}^{(1)}\left(q^{\prime} \sigma\right)}\right|^{2} \frac{-i}{\left(\omega+\omega^{\prime}\right)-i x}
$$

The first striking feature of eq. (66) is that, in the conformal case $\nu=1 / 2$, the result for radiative back reaction is identical to the flat space result. In the non-conformal case, we can obtain the large $\sigma x$ limit from the large argument expansion of the Hankel functions, for example

$$
\operatorname{Im} F_{5}(x) \sim-\frac{x^{6}}{16384 \pi^{2}} \ln \left(\frac{x}{\mu}\right)
$$

in five dimensions. The small $\sigma x$ limit can correspondingly be obtained from the small argument expansion of the Hankel functions, which leads to

$$
\operatorname{Im} F_{5}(x) \sim C x^{6}(\sigma x)^{4 \nu-2}
$$

when $\sigma x \ll 1$, where $C$ is a constant.

\section{EQUATIONS OF MOTION WITH LOGARITHMIC TERMS}

We can now construct the equation of motion for the brane. For a small perturbation $z$ of a flat brane, the scale factor $a \approx 1$ and $\partial_{t} z \approx-\sigma H$. For conformal scalar field radiation, eq. [67) suggests that eq. (86) would become

$$
\frac{d H}{d t}+2 H^{2}-2 H_{0}^{2}=-A \kappa_{5}^{2} \sigma \ln \left(\frac{1}{\mu} \frac{d}{d t}\right) \frac{d^{5} H}{d t^{5}} .
$$

where $H_{0}^{2}=\Lambda / 3$ and $A$ is a numerical coefficient. Strictly speaking, this equation is incomplete because there may be additional radiation damping terms of order $H^{2}$.

The radiation damping term calculated here is small in recent cosmological eras. Returning to eq (6), we see that the combination $\kappa_{5}^{2} \sigma \sim G \sigma^{2}$ in order of magnitude. The experimental lower bound on $\left(G \sigma^{2}\right)^{-1 / 4}$ is around $1 T e V$, but there is no reason for the the value to be much smaller than the usual Planck scale. When $\dot{H} \sim H^{2}$, the damping term is only important when $H^{4} \sim\left(G \sigma^{2}\right)^{-1}$. 
There is a small residual dark radiation effect given by eq. (10). If we take into account a period of inflation, ending at time $t_{I}$, then the ratio of dark radiation to ordinary radiation is

$$
6 A \kappa_{5}^{2} \sigma \int_{t_{I}}^{\infty} \frac{H}{\rho} \ln \left(\frac{1}{\mu} \frac{d}{d t}\right) \frac{d^{5} H}{d t^{5}} d t
$$

which is of order $G \sigma^{2} H\left(t_{I}\right)^{4}$. In principle, $H\left(t_{I}\right)$ should be close to the value of $H$ determined by the tensor perturbation amplitude $A_{T}$ in the cosmic microwave background, $A_{T}^{2}=8 \pi G H^{2}[28$. To be within nucleosynthesis constraints, the amount of dark radiation must be small [12, 16], and therefore there is a lower bound on the string vacuum energy scale of approximately

$$
\left(G \sigma^{2}\right)^{-1 / 4}>A_{T}^{-1 / 2} m_{p}
$$

where $m_{p}$ is the Planck mass.

We shall analyse a slightly more general type of equation of motion,

$$
\frac{d p}{d t}-F(p)=-\eta \ln \left(\frac{1}{\mu} \frac{d}{d t}\right) \frac{d^{n} p}{d t^{n}}
$$

A reasonable definition of the logarithmic term should be linear and causal, to ensure that the back reaction depends on the history of the source. We can begin be defining the action of the logarithmic derivative on exponentials,

$$
\ln \left(\frac{1}{\mu} \frac{d}{d t}\right) e^{\alpha t}=\ln \left(\frac{\alpha}{\mu}\right) e^{\alpha t}
$$

where we take $\alpha>0$. This leads to the following definition,

$$
\ln \left(\frac{1}{\mu} \frac{d}{d t}\right) f=-\int_{-\infty}^{t} \ln \left(e^{\gamma} \mu\left(t-t^{\prime}\right)\right) \frac{d f}{d t^{\prime}} d t^{\prime}
$$

where $\gamma$ is Euler's constant. The definition is linear, causal and correctly reproduces the action on exponentials. The integral can be evaluated for a broad class of functions, including discontinuous functions, for example

$$
\ln \left(\frac{1}{\mu} \frac{d}{d t}\right) \theta(t) e^{\alpha t}=\left(\mathrm{E}_{1}(\alpha t)+\ln \left(\frac{\alpha}{\mu}\right)\right) \theta(t) e^{\alpha t}
$$

where $\mathrm{E}_{1}$ is the exponential integral. If $f$ is integrable, then the logarithmic derivative of $f$ is $O\left(t^{-1}\right)$ as $t \rightarrow \infty$.

For a linear equation of motion (72), with $F=-\lambda p$, we have

$$
\frac{d p}{d t}+\lambda p=-\eta \ln \left(\frac{1}{\mu} \frac{d}{d t}\right) \frac{d^{5} p}{d t^{5}}
$$

The exponential solutions $\exp (\mu z t)$ satisfy

$$
z+\alpha=-\beta z^{n} \ln (z)
$$

where $\alpha=\mu^{-1} \lambda$ and $\beta=\eta \mu^{n-1}$. The roots for a sample case are plotted in figure 3 When there is no reaction term there is only a decaying mode corresponding to $z=-\alpha$. The reaction term shifts this solution and introduces an oscillation. There are two other decaying solutions and two growing modes. These modes have $|z|>1$ and damping or growth rates larger than the renormalisation scale $\mu$. We tentatively identify these as unphysical runaway solutions.

Runaway solutions can be excluded by replacing the equation of motion by an integrodifferential equation. First, we introduce a Green function which decays exponentially as the time $t \rightarrow \pm \infty$ and satisfies the following equation,

$$
G+\eta \ln \left(\frac{1}{\mu} \frac{d}{d t}\right) \frac{d^{n-1} G}{d t^{n-1}}=\delta\left(t-t^{\prime}\right)
$$

The equation of motion (72) can then be rewritten in an alternative form

$$
\frac{d p}{d t}=\int_{-\infty}^{\infty} G\left(t-t^{\prime}\right) F\left(t^{\prime}\right)
$$




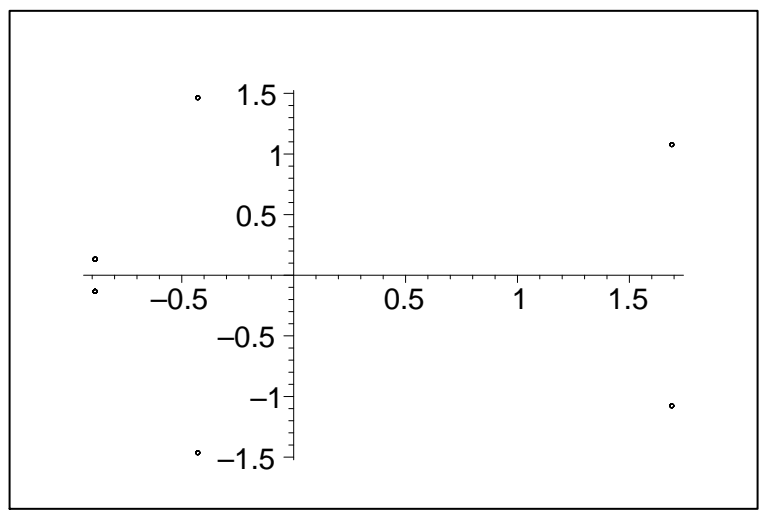

FIG. 3: The roots of $1+z=-0.1 z^{5} \ln (z)$ in the complex plane.

The runaway solutions are excluded at the expense of a preacceleration term, as we can see from the expression for the Green function

$$
G\left(t-t^{\prime}\right)=\sum_{\operatorname{Re} z_{i}<0} c_{i} e^{z_{i}\left(t-t^{\prime}\right)} \theta\left(t-t^{\prime}\right)-\sum_{\operatorname{Re} z_{i}>0} c_{i} e^{z_{i}\left(t-t^{\prime}\right)} \theta\left(t^{\prime}-t\right),
$$

where $z_{i}$ are the roots of $\eta z^{n-1} \ln (z / \mu)=-1$ and $c_{i}$ are constants fixed by continuity relations at $t=t^{\prime}$. The acausality is represented by the final term, but it is a relatively minor effect if $\operatorname{Re} z_{i} \gg \mu$, because then the acausality only occurs on timescales small compared to the renormalisation timescale.

An interesting variant of the equation of motion (72) occurs for the case $n=1$ and $F=0$. There are two real solutions, $p=0$ and $p=\exp \left(\exp \left(-\mu \eta^{-1}\right) t\right)$. Because of the small size of the exponent it would not be appropriate to regard the exponential solution as a runaway solution. In a cosmological context, a flat brane would spontaneously begin to accelerate with a tiny, positive cosmological constant. We have not been able to find a model with this particular radiation reaction so far.

\section{CONCLUSION}

This paper has been devoted to calculating the radiation back reaction forces on a moving brane. We have found that massless, conformally invariant scalar radiation into the bulk results in logarithmic terms in the equations of motion. The radiation reaction leads to both physical and runaway solutions. The runaway solutions can be excluded by introducing an integrodifferential formulation of the equation of motion. This form of the equation could be used if we were to attempt to solve the equations of motion numerically. The question of whether the brane's initial trajectory might be destabilised by radiation reaction forces remains an open one.

The radiation from the moving brane also leaves a residual effect in the form of dark radiation. The amount of this radiation depends on the brane trajectory (70). An order of magnitude estimate combined with a crude nucleosynthesis constaint gives a lower bound on the string vacuum energy scale of $\sqrt{A_{T}} m_{p}$, where $A_{T}$ is the tensor perturbation amplitude in the cosmic microwave background.

The radiation reaction problem has been set up in a way which can be generalised to non-scalar fields. One issue which would be of interest is the possibility of supersymetric cancellations amongst some of the terms. The regularisation methods used in this paper have enabled us to neglect the non-logarithmic divergences, but it would be better to see direct cancellation of these divergent terms. For broken supersymmetry, we expect that the renormalisation scale, which appears in our results, would be replaced by a supersymmetry breaking scale.

The results for the radiation reaction of branes moving in anti-de Sitter space generalises in a very staightforward way to any background where the modes of the radiation fields are known explicitly. An example of this is the low energy limit of the heterotic string [1, 29, 30], where the modes of the graviton multiplet are known and can be found in [31].

Another aspect of the radiation reaction which is worth further study is the effect of different higher dimensional vacuum states. These states can have thermal properties and the reaction forces would have similarities to those 
acting on moving mirrors at finite temperatures.

[1] P. Horava and E. Witten, Nucl. Phys. B475, 94 (1996).

[2] T. Shiromizu, K.-i. Maeda, and M. Sasaki, Phys. Rev. D62, 024012 (2000), gr-qc/9910076.

[3] J. M. Cline, C. Grojean, and G. Servant, Phys. Rev. Lett. 83, 4245 (1999), hep-ph/9906523.

[4] P. Binetruy, C. Deffayet, and D. Langlois, Nucl. Phys. B565, 269 (2000), hep-th/9905012.

[5] S. A. Fulling and P. C. W. Davies, Proc. Roy. Soc. Lond. A348, 393 (1976).

[6] L. H. Ford and A. Vilenkin, Phys. Rev. D25, 2569 (1982).

[7] M.-T. Jaekel and S. Reynaud, J. Phys. I(France) 3, 339 (1993), quant-ph/9801072.

[8] L. A. S. Machado, P. A. Maia Neto, and C. Farina, Phys. Rev. D66, 105016 (2002), hep-th/0207035.

[9] L. M. Landau and E. M. Lifshitz, The classical theory of fields (Pergamon, Oxford, 1971), 3rd ed.

[10] M. R. Setare (2004), hep-th/0406222.

[11] D. Langlois and L. Sorbo, Phys. Rev. D68, 084006 (2003), hep-th/0306281.

[12] A. Hebecker and J. March-Russell, Nucl. Phys. B608, 375 (2001), hep-ph/0103214.

[13] D. Langlois, L. Sorbo, and M. Rodriguez-Martinez, Phys. Rev. Lett. 89, 171301 (2002), hep-th/0206146.

[14] K. Enqvist, A. Mazumdar, and A. Perez-Lorenzana (2004), hep-th/0403044.

[15] P. Binetruy, C. Deffayet, U. Ellwanger, and D. Langlois, Phys. Lett. B477, 285 (2000), hep-th/9910219.

[16] E. E. Flanagan, S. H. H. Tye, and I. Wasserman, Phys. Rev. D62, 044039 (2000), hep-ph/9910498.

[17] N. D. Birrell and P. C. W. Davies, Quantum fields in curved space (Cambridge University Press, 1982).

[18] J. Schwinger, J Math Phys 2, 407 (1961).

[19] L. V. Keldysh, Zh. Eksp. Teor. Fiz. 47, 1515 (1964).

[20] E. Calzeta and B. L. Hu, Phys. Rev. D 35, 495 (1987).

[21] I. G. Moss, W. Naylor, W. Santiago-German, and M. Sasaki, Phys. Rev. D67, 125010 (2003), hep-th/0302143.

[22] G. W. Gibbons and E. P. S. Shellard, eds., The future of theoretical physics and cosmology: Celebrating Stephen Hawking's 60th birthday. Proceedings, Workshop and Symposium, Cambridge, UK, January 7-10, 2002 (Cambridge University Press, 2004).

[23] T. Vachaspati, A. E. Everett, and A. Vilenkin, Phys. Rev. D30, 2046 (1984).

[24] P. Kraus, JHEP 12, 011 (1999), hep-th/9910149.

[25] A. Kehagias and E. Kiritsis, JHEP 11, 022 (1999), hep-th/9910174.

[26] L. Randall and R. Sundrum, Phys. Rev. Lett. 83, 3370 (1999), hep-ph/9905221.

[27] L. Randall and R. Sundrum, Phys. Rev. Lett. 83, 4690 (1999), hep-th/9906064.

[28] A. R. Liddle and D. H. Lyth, Phys. Rept. 231, 1 (1993), astro-ph/9303019.

[29] A. Lukas, B. A. Ovrut, K. S. Stelle, and D. Waldram, Phys Rev D 59, 086001 (1999).

[30] A. Lukas, B. A. Ovrut, K. S. Stelle, and D. Waldram, Nucl Phys B 552, 246 (1999).

[31] I. G. Moss and J. P. Norman (2004), hep-th/0401181. 